\title{
Bipolarons and multipolarons consisting of impurity atoms in a Bose-Einstein condensate
}

\author{
W. Casteels, ${ }^{1}$ J. Tempere, ${ }^{1,2}$ and J. T. Devreese ${ }^{1}$ \\ ${ }^{1}$ TQC, Universiteit Antwerpen, Universiteitsplein 1, 2610 Wilrijk, Belgium \\ ${ }^{2}$ Lyman Laboratory of Physics, Harvard University, Cambridge, Massachusetts 02138, USA
}

(Received 12 April 2013; published 9 July 2013)

\begin{abstract}
The variational Feynman formalism for the polaron, extended to an all-coupling treatment of bipolarons, is applied for two impurity atoms in a Bose-Einstein condensate. This shows that if the polaronic coupling strength is large enough, the impurities will form a bound state (the bipolaron). As a function of the mutual repulsion between the impurities, two types of bipolaron are distinguished: a tightly bound bipolaron at weak repulsion and a dumbbell bipolaron at strong repulsion. Apart from the binding energy, the evolution of the bipolaron radius and its effective mass are also examined as a function of the strength of the repulsive interaction between the impurities and of the polaronic coupling strength. We then apply the strong-coupling formalism to multiple-impurity atoms in a condensate, which leads to the prediction of multipolaron formation in the strong-coupling regime. The results of the two formalisms are compared for two impurities in a condensate, which results in a general qualitative agreement and a quantitative agreement at strong coupling. Typically, the system of impurity atoms in a Bose-Einstein condensate is expected to exhibit the polaronic weak-coupling regime. However, the polaronic coupling strength is, in principle, tunable with a Feshbach resonance.
\end{abstract}

DOI: 10.1103/PhysRevA.88.013613

PACS number(s): 67.85.Bc, 63.20.kd

\section{INTRODUCTION}

In recent years systems related to ultracold gases have successfully been applied as quantum simulators for many-body theories [1]. A specific example is a Bose-Einstein condensate (BEC) with an impurity atom of which the Hamiltonian can be mapped onto the Fröhlich polaron Hamiltonian, provided the Bogoliubov approximation is valid [2-4]. The polaron is a well-known concept in solid-state physics, where it represents the quasiparticle that consists of an electron in a polar or ionic lattice, dressed with the self-induced polarization cloud, which is described by the lattice vibrations or phonons [5]. In the context of ultracold atomic gases the electron is replaced by an impurity atom, and the role of the phonons is played by the Bogoliubov excitations of the condensate. Hitherto, the Fröhlich Hamiltonian has resisted analytical diagonalization, making it the subject of various approximation schemes [6]. A polaronic system typically exhibits different coupling regimes characterized by a quasifree polaron at weak coupling and a bound state in the self-induced potential at strong coupling. The variational all-coupling treatment, as developed by Feynman to describe the ground state of the polaron [7], reveals the transition between the coupling regimes and nicely interpolates between the Fröhlich perturbative result at weak coupling [8] and the Landau-Pekar strong-coupling result $[9,10]$. For a single impurity in a Bose-Einstein condensate the application of the Feynman all-coupling theory also revealed the transition between the different coupling regimes [11,12].

Recently, there have been reports on the immersion of a single impurity in an ultracold gas [13-17]. For neutral impurity atoms in a BEC the system is expected to be in the weak polaronic coupling regime. For a ${ }^{6} \mathrm{Li}$ impurity in a $\mathrm{Na}$ condensate, for example, the polaronic coupling strength $\alpha$ is of the order of $10^{-3}$ [11], which is well within the weak-coupling regime. However, the polaronic coupling strength is, in principle, externally tunable and can be increased by means of a Feshbach resonance. The feasibility of interspecies Feshbach resonances has recently been demonstrated in various ultracold mixtures (see, for example, Refs. [18-21]). An experimental study of the BEC impurity polaron with a variable coupling strength could shed light on discrepancies between different predictions of the polaronic dynamic response properties [22-24].

Considering multiple impurities in a Bose-Einstein condensate unveils a whole range of interesting phenomena. The presence of the Bose-Einstein condensate induces an effective interaction between the impurities [25,26]. At weak polaronic coupling the application of the many-polaron formalism, as developed in Refs. [27,28], leads to a description of the groundstate properties and the response to Bragg spectroscopy of ultracold weakly interacting binary mixtures [29]. If the polaronic coupling is strong enough, the BEC-induced interaction can lead to the clustering of the impurities, also known as a multipolaron [30,31]. The possibility of the formation of a multipolaron at strong polaronic coupling is also well known in the solid-state context [32-36]. A special case is the formation of a bound state of two electrons, commonly known as the bipolaron [37-39]. Bipolarons have attracted much attention because of their possible role as an unconventional pairing mechanism for high-temperature superconductivity [40,41].

In this work, we consider a few impurities in a BoseEinstein condensate and examine the formation of a multipolaron. We start in Sec. II by showing how the Hamiltonian of impurity atoms in a BEC can be mapped onto the Fröhlich Hamiltonian, provided the Bogoliubov approximation is valid. Then, in Sec. III, the all-coupling variational path-integral approach, as developed by Feynman in Ref. [7] for a single polaron and extended in Ref. [42] for two electrons in a polar or ionic lattice, is applied to two distinguishable impurities in a condensate. This allows us to examine the polaron-bipolaron transition and the bipolaron ground-state properties such as the radius and the effective mass. In Sec. IV a strong-coupling treatment for multiple impurities in a BEC, based on the Landau-Pekar strong-coupling approach, is considered. This is first applied for two impurities in a condensate to examine the 
polaron-bipolaron transition and the polaronic properties and then to multiple impurities to study the formation of a larger multipolaron. Finally, in Sec. V, we compare the results of the two presented formalisms for two distinguishable impurities in a BEC, and in Sec. VI we conclude.

\section{THE POLARONIC SYSTEM CONSISTING OF MULTIPLE IMPURITIES IN A BOSE-EINSTEIN CONDENSATE}

The Hamiltonian of $N_{I}$ impurity atoms, in the presence of a homogeneous Bose gas, is given by

$$
\begin{aligned}
\hat{H}= & \sum_{i=1}^{N_{I}} \frac{\hat{p}_{i}^{2}}{2 m_{I}}+\sum_{\vec{k}} E_{\vec{k}} \hat{a}_{\vec{k}}^{\dagger} \hat{a}_{\vec{k}}+\frac{1}{2} \sum_{\vec{k}, \vec{k}^{\prime}, \vec{q}} V_{B B}(\vec{q}) \hat{a}_{\vec{k}^{\prime}-\vec{q}}^{\dagger} \hat{a}_{\vec{k}+\vec{q}}^{\dagger} \hat{a}_{\vec{k}} \hat{a}_{\vec{k}^{\prime}} \\
& +\sum_{\vec{k}, \vec{q}} V_{I B}(\vec{q}) \hat{\rho}_{I}(\vec{q}) \hat{a}_{\vec{k}-\vec{q}}^{\dagger} \hat{a}_{\vec{k}}+\sum_{i<j}^{N_{I}} V_{I I}\left(\hat{\vec{r}}_{i}-\hat{\vec{r}}_{j}\right) .
\end{aligned}
$$

The first term represents the kinetic energy of the impurities of mass $m_{I}$ and associated momentum operators $\left\{\hat{\vec{p}}_{i}\right\}$ and position operators $\left\{\hat{\vec{r}}_{i}\right\}$. The operator $\hat{a}_{\vec{k}}^{\dagger}\left(\hat{a}_{\vec{k}}\right)$ creates (annihilates) a boson with mass $m_{B}$, wave vector $\vec{k}$, and energy $E_{\vec{k}}=$ $(\hbar k)^{2} / 2 m_{B}-\mu$, with $\mu$ being the boson chemical potential. These bosons interact mutually, with $V_{B B}(\vec{q})$ being the Fourier transform of the interaction potential. The Fourier transform of the impurity-boson interaction potential is $V_{I B}(\vec{q})$ and couples the boson density to the impurity density

$$
\hat{\rho}_{I}(\vec{q})=\sum_{i=1}^{N_{I}} e^{i \vec{q} \cdot \hat{\vec{r}}_{i}}
$$

The impurity-impurity interaction is described by the interaction potential $V_{I I}(\vec{r})$. For the interparticle interactions we assume contact pseudopotentials: $V_{B B}(\vec{r})=g_{B B} \delta(\vec{r}), V_{I B}(\vec{r})=$ $g_{I B} \delta(\vec{r})$, and $V_{I I}(\vec{r})=g_{I I} \delta(\vec{r})$. The interaction strengths $g_{B B}$, $g_{I B}$, and $g_{I I}$ are related to the corresponding scattering lengths $a_{B B}, a_{I B}$, and $a_{I I}$ through the Lippmann-Schwinger equation. For the boson-boson and the impurity-impurity interactions the first-order result suffices: $g_{B B}=4 \pi \hbar^{2} a_{B B} / m_{B}$ and $g_{I I}=$ $4 \pi \hbar^{2} a_{I I} / m_{I}$. We will only consider repulsive impurities $\left(a_{I I}>0\right)$. For the impurity-boson interaction the LippmannSchwinger equation has to be treated up to second order to obtain convergent final results:

$$
\frac{2 \pi \hbar^{2} a_{I B}}{m_{r}}=g_{I B}-g_{I B}^{2} \sum_{\vec{k} \neq 0} \frac{2 m_{r}}{(\hbar k)^{2}},
$$

where $m_{r}$ is the reduced mass $\left(m_{r}^{-1}=m_{I}^{-1}+m_{B}^{-1}\right)$.

If a Bose-Einstein condensate is realized, the number of bosons $N_{0}$ that occupy the single-particle ground state becomes a macroscopic number: $N_{0} \gg 1$ [43]. This is expressed by the Bogoliubov shift [44], which transforms Hamiltonian (1) into

$$
\hat{H}=E_{G P}+N_{I} N_{0} g_{I B}+\hat{H}_{\mathrm{pol}}^{\left(N_{I}\right)},
$$

where $E_{G P}$ is the Gross-Pitaevskii energy of the homogeneous condensate $[45,46]$. The second term in Hamiltonian (4) is the interaction shift due to the impurities, and the third term is the
Fröhlich Hamiltonian for $N_{I}$ polarons:

$$
\begin{aligned}
\hat{H}_{\mathrm{pol}}^{\left(N_{I}\right)}= & \sum_{i=1}^{N_{I}} \frac{\hat{p}_{i}^{2}}{2 m_{I}}+\sum_{\vec{k}} \hbar \omega_{\vec{k}} \hat{\alpha}_{\vec{k}}^{\dagger} \hat{\alpha}_{\vec{k}}+\sum_{\vec{k}} V_{\vec{k}} \hat{\rho}_{I}(\vec{q})\left(\hat{\alpha}_{\vec{k}}+\hat{\alpha}_{-\vec{k}}^{\dagger}\right) \\
& +\sum_{i<j}^{N_{I}} V_{I I}\left(\hat{\vec{r}}_{i}-\hat{\vec{r}}_{j}\right) .
\end{aligned}
$$

The first term of the Fröhlich Hamiltonian represents the kinetic energy of the impurities. The operator $\hat{\alpha}_{\vec{k}}^{\dagger}\left(\hat{\alpha}_{\vec{k}}\right)$ creates (annihilates) a Bogoliubov excitation with wave number $\vec{k}$ and energy

$$
\hbar \omega_{\vec{k}}=\frac{\hbar^{2} k}{2 m_{B} \xi} \sqrt{2+(\xi k)^{2}},
$$

where the healing length of the Bose condensate was introduced: $\xi=1 / \sqrt{8 \pi a_{B B} n_{0}}$, with $n_{0}=N_{0} / V$ being the condensate density (we work with unit volume for the homogenous gas). The third term in the Fröhlich Hamiltonian (5) describes the interaction between the impurities and the Bogoliubov excitations with the interaction amplitude

$$
V_{\vec{k}}=\sqrt{N_{0}} g_{I B}\left[\frac{(\xi k)^{2}}{(\xi k)^{2}+2}\right]^{1 / 4} .
$$

The last term of the Fröhlich Hamiltonian (5) corresponds to the interaction between the impurities. The Fröhlich Hamiltonian (5) was originally derived to describe the interaction between an electron (or hole) and the longitudinal optical phonons in a polar or ionic crystal [8]. A strong repulsion between the impurity and the bosons can lead to a large depletion of the condensate in the vicinity of the impurity, which can break the validity of the Bogoliubov approximation and the applicability of the Fröhlich Hamiltonian to describe the system. This typically leads to a bubble state [47].

\section{ALL-COUPLING VARIATIONAL TREATMENT FOR TWO IMPURITY ATOMS IN A BEC}

\section{A. All-coupling formalism}

We consider the generic polaronic system of two distinguishable particles interacting with a bosonic bath through the Fröhlich interaction, i.e., any system that can be described by Hamiltonian (5) with $N_{I}=2$. The variational all-coupling single-polaron treatment, as originally developed by Feynman [7], was extended in Ref. [42] to the case of two polaronic particles. This approach is based on the Jensen-Feynman variational inequality for the free energy $\mathcal{F}[48,49]$ :

$$
\mathcal{F} \leqslant \mathcal{F}_{0}+\frac{1}{\hbar \beta}\left\langle\mathcal{S}-\mathcal{S}_{0}\right\rangle_{\mathcal{S}_{0}}
$$

where $\mathcal{S}$ is the action of the polaronic system as described by the Fröhlich Hamiltonian $(5), \mathcal{S}_{0}$ is the action of a variational trial system with free energy $\mathcal{F}_{0}$, and $\beta$ is the inverse temperature $T: \beta=\left(k_{B} T\right)^{-1}$. Eliminating the degrees of freedom of the Bogoliubov excitations leads to an effective 


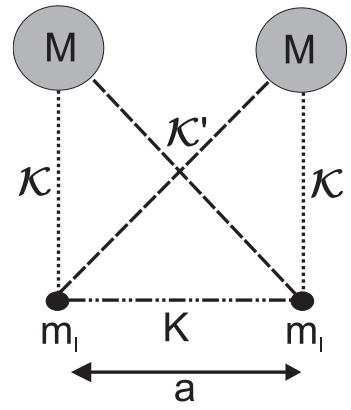

FIG. 1. Schematic picture of the variational trial system for two polaronic particles, as introduced in Ref. [42]. The black dots represent the impurity atoms of mass $m_{I}$, and the larger gray dots depict the fictitious particles of mass $M$. The connecting lines represent harmonic interactions with the corresponding oscillator strengths indicated.

polaron action containing retardation effects:

$$
\begin{aligned}
\mathcal{S}= & \int_{0}^{\hbar \beta} d \tau\left[\sum_{i=1}^{N_{I}} \frac{m_{I}}{2} \dot{r}_{i}^{2}(\tau)+\sum_{i<j} V_{I I}\left(\vec{r}_{i}-\vec{r}_{j}\right)\right] \\
& -\sum_{j, l=1}^{N_{I}} \sum_{\vec{k}} \frac{\left|V_{\vec{k}}\right|^{2}}{\hbar} \int_{0}^{\hbar \beta} d \tau \\
& \times \int_{0}^{\hbar \beta} d \sigma G_{\omega_{\vec{k}}}(\tau-\sigma) e^{i \vec{k} \cdot\left[\vec{r}_{j}(\tau)-\vec{r}_{l}(\sigma)\right]},
\end{aligned}
$$

with $G_{\omega_{\vec{k}}}(u)$ being the Green's function of the Bogoliubov excitations:

$$
G_{\omega_{\vec{k}}}(u)=\frac{\cosh \left[\omega_{\vec{k}}(\hbar \beta / 2-|u|)\right]}{2 \sinh \left(\beta \hbar \omega_{\vec{k}} / 2\right)} .
$$

For a single polaron a variational system was suggested by Feynman that mimics the influence of the interaction with the Bogoliubov excitations on the impurity by a harmonic coupling to a fictitious particle of mass $M$ with oscillator strength $\kappa$ [7]. The upper bound for the free energy (8) is then minimized as a function of the variational parameters $M$ and $\kappa$. In Ref. [42] an extension of this trial system was introduced for the case of two polaronic particles, which is schematically presented in Fig. 1. As is the case in the Feynman one-polaron trial system the impurities interact quadratically with fictitious particles of mass $M$ with oscillator strength $\kappa$. Furthermore, there is a quadratic interaction, with oscillator strength $\kappa^{\prime}$, with the fictitious particle of the other impurity. The particles are separated by the vector $\vec{a}$, and they mutually interact quadratically with strength $K$. For $\kappa^{\prime}=K=0$ this reduces to (twice) the Feynman model system. After transforming to the eigenmodes the action of the trial system can be written as

$$
\mathcal{S}_{0}=\int_{0}^{\hbar \beta} d \tau\left[\frac{\mu_{0}}{2} \dot{\rho}_{0}^{2}+\sum_{j=1}^{3}\left(\frac{\mu_{j}}{2} \dot{\rho}_{j}^{2}+\frac{1}{2} \mu_{j} \Omega_{j}^{2} \rho_{j}^{2}\right)\right],
$$

with $\left\{\vec{\rho}_{i}\right\}$ being the coordinates of the eigenmodes of the trial system and $\left\{\Omega_{i}\right\}$ being the corresponding eigenfrequencies $\left(\Omega_{0}=0\right.$ corresponds to a translation of the trial system as

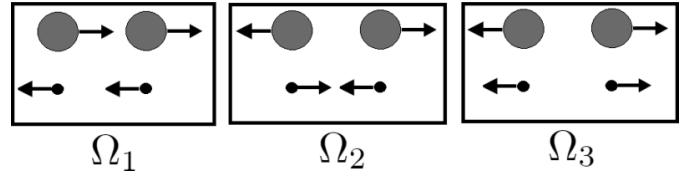

FIG. 2. Schematic presentation of the eigenmodes corresponding to the eigenfrequencies $\Omega_{1}, \Omega_{2}$, and $\Omega_{3}$. The small black dots represent the impurities, and the larger gray dots depict the fictitious particles of mass $M$.

a whole):

$$
\begin{aligned}
\Omega_{1}^{2}=\frac{M+m_{I}}{M m_{I}}\left(\kappa+\kappa^{\prime}\right), & \\
\Omega_{2,3}^{2}= & \frac{1}{2}\left\{\frac{M+m_{I}}{M m_{I}}\left(\kappa+\kappa^{\prime}\right)-\frac{2 K}{m_{I}}\right. \\
& \pm \sqrt{\left.\left[\frac{M-m_{I}}{M m_{I}}\left(\kappa+\kappa^{\prime}\right)-\frac{2 K}{m_{I}}\right]^{2}+\frac{4}{m_{I} M}\left(\kappa-\kappa^{\prime}\right)^{2}\right\}} .
\end{aligned}
$$

Since all oscillator strengths are positive, the eigenfrequencies satisfy the inequalities

$$
\begin{gathered}
\Omega_{1}^{2} \geqslant \Omega_{2}^{2}+\Omega_{3}^{2}, \\
\Omega_{2} \geqslant v \geqslant \Omega_{3} \geqslant 0,
\end{gathered}
$$

where we have introduced the frequency parameter $v=$ $\sqrt{\left(\kappa+\kappa^{\prime}\right) / M}$. In expression (11) the following mass factors were introduced:

$$
\begin{aligned}
& \mu_{0}=2\left(m_{I}+M\right), \quad \mu_{2}=\frac{2 m_{I} M}{\left(m_{I}+M\right)}, \\
& \mu_{2}=1, \quad \mu_{3}=1 .
\end{aligned}
$$

The corresponding eigenmodes of the trial system are schematically presented in Fig. 2. The action (11) shows that the trial system decouples into a free particle and three harmonic oscillators.

After eliminating the degrees of freedom of the two fictitious particles of mass $M$ the effective action of the trial system becomes retarded and can be written as

$$
\begin{aligned}
\mathcal{S}_{0}= & \int_{0}^{\hbar \beta} d \tau\left[\sum_{i=1,2} \frac{m_{I}}{2} \dot{r}_{i}^{2}(\tau)-\frac{K}{2}\left(\vec{r}_{1}-\vec{r}_{2}-\vec{a}\right)^{2}\right] \\
& +\frac{\kappa^{2}+\kappa^{\prime 2}}{4 M \nu} \int_{0}^{\hbar \beta} d \tau \int_{0}^{\hbar \beta} d \sigma G_{v}(\tau-\sigma) \\
& \times \sum_{j}\left[\vec{r}_{j}(\tau)-\vec{r}_{j}(\sigma)\right]^{2}+\frac{\kappa \kappa^{\prime}}{M \nu} \int_{0}^{\hbar \beta} d \tau \\
& \times \int_{0}^{\hbar \beta} d \sigma G_{v}(\tau-\sigma)\left[\vec{r}_{1}(\tau)-\vec{r}_{2}(\sigma)-\vec{a}\right]^{2} .
\end{aligned}
$$

Applying the Jensen-Feynman inequality then results in an upper bound $E$ for the polaronic contribution to the 
ground-state energy at zero temperature [42]:

$$
\begin{aligned}
E= & \sum_{j=1}^{3} \frac{3}{2} \hbar \Omega_{j}-3 \hbar v+\sum_{\vec{k}} V_{I I}(\vec{k}) e^{i \vec{k} \cdot \vec{a}} e^{-k^{2} D_{12}(0)} \\
& -2 \sum_{\vec{k}} \frac{\left|V_{\vec{k}}\right|^{2}}{\hbar} \int_{0}^{\infty} d u e^{-\omega_{\vec{k}} u}\left[e^{-k^{2} D_{11}(u)}+e^{i \vec{k} \cdot \vec{a}} e^{-k^{2} D_{12}(u)}\right] \\
& -\frac{3}{2} \frac{\Omega_{1}^{2}-v^{2}}{\Omega_{1}^{2}} \frac{\hbar \Omega_{1}}{2}-\frac{3}{2} \frac{\Omega_{2}^{2}-v^{2}}{\Omega_{2}^{2}-\Omega_{3}^{2}} \frac{\hbar \Omega_{2}}{2}-\frac{3}{2} \frac{v^{2}-\Omega_{3}^{2}}{\Omega_{2}^{2}-\Omega_{3}^{2}} \frac{\hbar \Omega_{3}}{2},
\end{aligned}
$$

where the functions $D_{11}(u)$ and $D_{12}(u)$ are defined as

$$
\begin{aligned}
D_{11}(u)= & \frac{\hbar}{2 m_{I}}\left[\frac{v^{2}}{\Omega_{1}^{2}} \frac{u}{2}+\frac{\Omega_{1}^{2}-v^{2}}{\Omega_{1}^{2}} E\left(\Omega_{1}, u\right)\right. \\
& \left.+\frac{\Omega_{2}^{2}-v^{2}}{\Omega_{2}^{2}-\Omega_{3}^{2}} E\left(\Omega_{2}, u\right)+\frac{v^{2}-\Omega_{3}^{2}}{\Omega_{2}^{2}-\Omega_{3}^{2}} E\left(\Omega_{3}, u\right)\right], \\
D_{12}(u)= & \frac{\hbar}{2 m_{I}}\left[\frac{v^{2}}{\Omega_{1}^{2}} \frac{u}{2}+\frac{\Omega_{1}^{2}-v^{2}}{\Omega_{1}^{2}} E\left(\Omega_{1}, u\right)\right. \\
& \left.+\frac{\Omega_{2}^{2}-v^{2}}{\Omega_{2}^{2}-\Omega_{3}^{2}} F\left(\Omega_{2}, u\right)+\frac{v^{2}-\Omega_{3}^{2}}{\Omega_{2}^{2}-\Omega_{3}^{2}} F\left(\Omega_{3}, u\right)\right],
\end{aligned}
$$

with

$$
\begin{aligned}
& E(\Omega, u)=\frac{1-\exp [-\Omega u]}{2 \Omega}, \\
& F(\Omega, u)=\frac{1+\exp [-\Omega u]}{2 \Omega} .
\end{aligned}
$$

The next step is to minimize the upper bound $E$ (18) as a function of the variational parameters $\left\{v, \Omega_{1}, \Omega_{2}, \Omega_{3}, \vec{a}\right\}$.

\section{Bipolaron radius}

As an estimate for the bipolaron radius $R$ the root mean square of the distance between the impurities is used [42]:

$$
\begin{aligned}
R & =\sqrt{\left\langle\left[\vec{r}_{1}(\tau)-\vec{r}_{2}(\tau)\right]^{2}\right\rangle} \\
& =\sqrt{a^{2}+6 D_{12}(0)},
\end{aligned}
$$

with the function $D_{12}(u)$ as defined in (20).

\section{Effective mass of the bipolaron}

The effective mass $m^{*}$ can be derived from the path-integral propagation from $\vec{r}_{i}(0)$ to $\vec{r}_{i}(T)=\vec{r}_{i}(0)+\vec{U} T$ for $i=1,2$. The ground-state energy then behaves as

$$
E(U)=E(0)+\frac{m^{*} U^{2}}{2} .
$$

This procedure was implemented by Feynman to derive the effective mass of a single polaron at arbitrary coupling [7]. The same treatment for two particles leads to an expression for the effective mass of the bipolaron:

$$
\begin{aligned}
m^{*}= & 2 m_{I}+2 \sum_{\vec{k}} \frac{\left|V_{\vec{k}}\right|^{2}}{\hbar} \\
& \times \int_{0}^{\infty} d u e^{-\omega_{\vec{k}} u}\left[e^{-k^{2} D_{11}(u)}+e^{i \vec{k} \cdot \vec{a}} e^{-k^{2} D_{12}(u)}\right] u^{2} k_{z}^{2},
\end{aligned}
$$

with functions $D_{11}(u)$ and $D_{12}(u)$ as defined in (19) and (20), respectively.

\section{Single-polaron limit}

The trial system reduces to (twice) the Feynman onepolaron trial system for $K=\kappa^{\prime}=0$. For the eigenmodes this corresponds to $\Omega_{3}=0$ and $\Omega_{1}=\Omega_{2}=\Omega$. This gives, for functions $D_{11}(u)$ [Eq. (19)] and $D_{12}(u)$ [Eq. (20)],

$$
\begin{aligned}
\lim _{\substack{\Omega_{3} \rightarrow 0 \\
\Omega_{1}=\Omega_{2}=\Omega}} D_{11}(u) & =\frac{\hbar}{2 m_{I}}\left[\frac{v^{2}}{\Omega^{2}} u+\frac{\Omega^{2}-v^{2}}{\Omega^{2}} \frac{1-\exp [-\Omega u]}{\Omega}\right] \\
& =D(u) \\
& \lim _{\substack{\Omega_{3} \rightarrow 0 \\
\Omega_{1}=\Omega_{2}=\Omega}} D_{12}(u) \rightarrow \infty
\end{aligned}
$$

The upper bound for the ground-state polaron energy (18) becomes in this limit

$$
\begin{aligned}
\lim _{\substack{\Omega_{3} \rightarrow 0 \\
\Omega_{1}=\Omega_{2}=\Omega}} E= & 2\left[\frac{3}{2} \hbar(\Omega-v)-\frac{3}{4} \frac{\Omega^{2}-v^{2}}{\Omega^{2}} \hbar \Omega\right. \\
& \left.-\sum_{\vec{k}} \frac{\left|V_{\vec{k}}\right|^{2}}{\hbar} \int_{0}^{\infty} d u e^{-\omega_{\vec{k}} u} e^{-k^{2} D(u)}\right] .
\end{aligned}
$$

This is (twice) the upper bound for the ground state of a single polaron, as derived by Feynman [7]. The effective mass of the bipolaron becomes in this limit

$$
\begin{aligned}
& \lim _{\substack{\Omega_{3} \rightarrow 0 \\
\Omega_{1}=\Omega_{2}=\Omega}} m^{*} \\
& =2\left(m_{I}+\frac{1}{3} \sum_{\vec{k}} \frac{\left|V_{\vec{k}}\right|^{2}}{\hbar} \int_{0}^{\infty} d u e^{-\omega_{\vec{k}} u} e^{-k^{2} D(u)} u^{2} k^{2}\right),
\end{aligned}
$$

which is (twice) the single-polaron effective mass, as derived by Feynman [7].

\section{B. Two impurities in a Bose-Einstein condensate}

We now consider the specific system of two impurity atoms in a Bose-Einstein condensate. Using the contact pseudopotential with the first-order Lippmann-Schwinger result gives for the impurity-impurity interaction

$$
\sum_{\vec{k}} V_{I I}(\vec{k}) e^{i \vec{k} \cdot \vec{a}} e^{-k^{2} D_{12}(0)}=\frac{\hbar^{2} a_{I I}}{2 \sqrt{\pi} m_{I} D_{12}(0)^{3 / 2}} e^{-\frac{a^{2}}{4 D_{12}(0)}} .
$$


Introducing the interaction amplitude (7) gives, for the upper bound for the ground-state energy (18), in polaronic units $\left(\hbar=m_{I}=\xi=1\right)$,

$$
\begin{aligned}
E= & \sum_{j=1}^{3} \frac{3}{2} \Omega_{j}-3 v+\frac{a_{I I}}{2 \sqrt{\pi} D_{12}(0)^{3 / 2}} e^{-\frac{a^{2}}{4 D_{12}(0)}} \\
& +\frac{\alpha}{2 \pi}\left(\frac{m_{B}+1}{m_{B}}\right)^{2} \int_{0}^{\infty} d k\left\{\frac{2 m_{B}}{m_{B}+1}-\frac{k^{3}}{\sqrt{k^{2}+2}}\right. \\
& \left.\times \int_{0}^{\infty} d u e^{-\omega_{\vec{k}} u}\left[e^{-k^{2} D_{11}(u)}+\frac{\sin [a k]}{a k} e^{-k^{2} D_{12}(u)}\right]\right\} \\
& -\frac{3}{2} \frac{\Omega_{1}^{2}-v^{2}}{\Omega_{1}^{2}} \frac{\Omega_{1}}{2}-\frac{3}{2} \frac{\Omega_{2}^{2}-v^{2}}{\Omega_{2}^{2}-\Omega_{3}^{2}} \frac{\Omega_{2}}{2}-\frac{3}{2} \frac{v^{2}-\Omega_{3}^{2}}{\Omega_{2}^{2}-\Omega_{3}^{2}} \frac{\Omega_{3}}{2},
\end{aligned}
$$

where $\alpha$ is the dimensionless polaronic coupling parameter:

$$
\alpha=\frac{a_{I B}^{2}}{\xi a_{B B}} .
$$

The first term in the $k$ integrand on the right-hand side of expression (31) is a consequence of using the LippmannSchwinger equation up to second order for the impurityboson interaction strength (3) in the second term of the total Hamiltonian (4) and is needed for convergence. A similar procedure was applied for the single-polaron all-coupling treatment in Ref. [11]. With the interaction amplitude (7) the bipolaron effective mass in polaronic units becomes

$$
\begin{aligned}
m^{*}= & +\frac{\alpha}{8 \pi^{2}}\left(\frac{1+m_{B}}{m_{B}}\right)^{2} \int d \vec{k} \sqrt{\frac{k^{2}}{k^{2}+2}} \\
& \times \int_{0}^{\infty} d u e^{-\omega_{\vec{k}} u}\left[e^{-k^{2} D_{11}(u)}+e^{i \vec{k} \cdot \vec{a}} e^{-k^{2} D_{12}(u)}\right] u^{2} k_{z}^{2} .
\end{aligned}
$$

\section{Results and discussion}

For numerical calculations it is favorable to introduce a cutoff $K_{c}$ for the $k$ integral in (31). Similar to the one-polaron case, we use the inverse of the van der Waals radius of the impurity-boson interaction potential for $K_{c}$ [11]. We introduce the specific system of ${ }^{6} \mathrm{Li}$ impurities in a Na condensate which amounts to $m_{B} / m_{I}=3.8227$ and $\xi K_{c}=200$. The considered system with two distinguishable impurities can, for example, be realized with two different hyperfine states of the same atom.

\section{Phase diagram}

The upper bound for the ground-state energy (31) was minimized as a function of the variational parameters $\left\{\vec{a}, \Omega_{1}, \Omega_{2}, \Omega_{3}, v\right\}$ for given values of the coupling parameter $\alpha$ and the impurity-impurity scattering length $a_{I I}$. If the resulting upper bound is lower than twice the upper bound for the one-polaron ground-state energy, we conclude that it is energetically favorable to form a bipolaron; otherwise, the system consists of two separate polarons. This procedure results in the $\left(a_{I I}, \alpha\right)$-phase diagram presented in Fig. 3, where we have also indicated three regions as a function of $\alpha$. For $\alpha>2.71$ the formation of a bipolaron is always

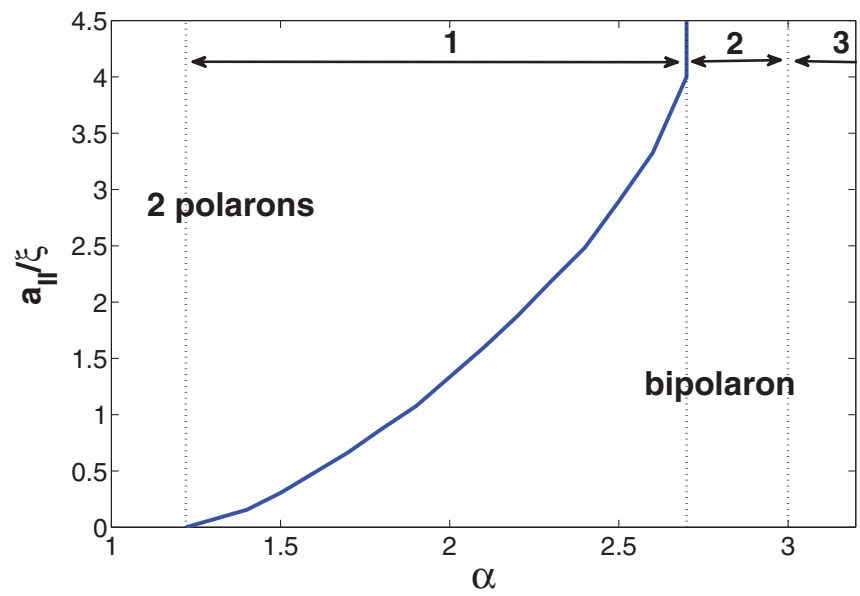

FIG. 3. (Color online) The $\left(a_{I I}, \alpha\right)$-phase diagram for two ${ }^{6} \mathrm{Li}$ impurities in a $\mathrm{Na}$ condensate with $\alpha$ being the polaronic coupling parameter and $a_{I I}$ being the impurity-impurity scattering length. The solid line indicates the polaron-bipolaron transition. The dotted lines at $\alpha=1.22, \alpha=2.71$, and $\alpha=3$ indicate the boundaries of the different regions, as discussed in the text.

energetically favorable, irrespective of $a_{I I}$, and the area with $\alpha \in[2.71,3]$ is denoted as region 2 (see Fig. 3). At $\alpha=3$ the Feynman all-coupling single-polaron treatment predicts the transition to the strong-coupling regime [11], and the area with $\alpha>3$ is denoted as region 3. From Fig. 3 it is clear that for $\alpha \in[1.22,2.71]$ (region 1) the bipolaron is only stable at small values of $a_{I I}$. For $\alpha<1.22$ a bipolaron is never formed. If the bipolaron is stable, the variationally determined vector $\vec{a}$, separating the impurities in the trial system of Fig. 1, is always zero in region 1 , irrespective of $a_{I I}$, while for $\alpha>2.71$ it is finite at sufficiently large $a_{I I}$, in which case the shape of the bipolaron can be interpreted as a dumbbell.

\section{Bipolaron mass and radius}

In Fig. 4 the upper bound (31), the polaronic effective mass (33), and the inverse bipolaron radius (23) are presented as
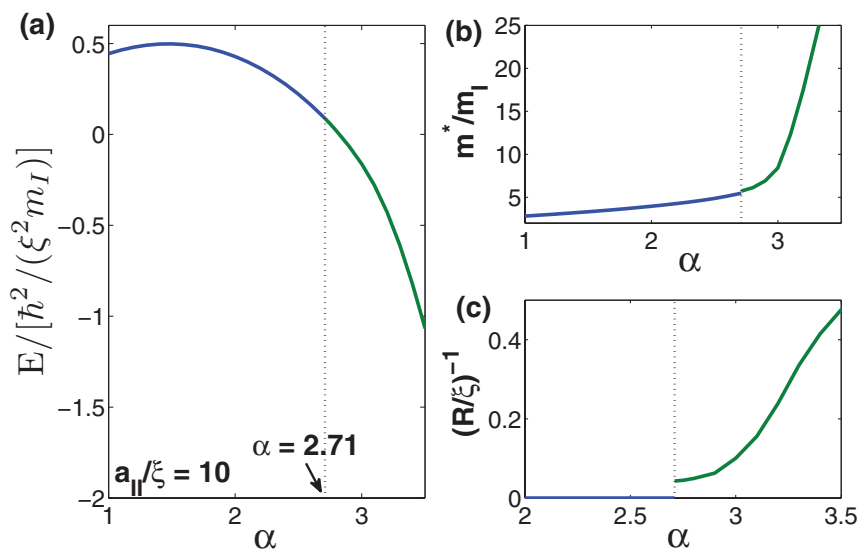

FIG. 4. (Color online) (a) The upper bound of the ground-state energy, (b) the bipolaron effective mass, and (c) the inverse of the bipolaron radius are presented as a function of the polaronic coupling parameter $\alpha$ at $a_{I I}=10$ for two ${ }^{6} \mathrm{Li}$ impurities in a Na condensate. The dotted line at $\alpha=2.71$ indicates the polaron-bipolaron transition. 


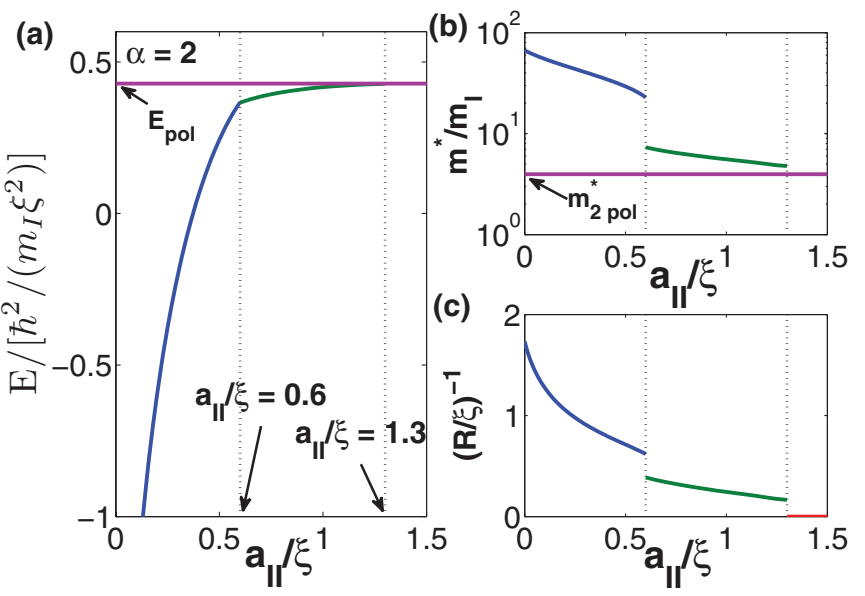

FIG. 5. (Color online) (a) The upper bound of the ground-state energy, (b) the effective mass (note the semilogarithmic scale), and (c) the inverse of the bipolaron radius are presented as a function of the impurity-impurity scattering length $a_{I I}$ at $\alpha=2$ (region 1 in Fig. 3) for two ${ }^{6} \mathrm{Li}$ impurities in a $\mathrm{Na}$ condensate. The polaron-bipolaron transition at $a_{I I} / \xi=1.3$ and the transition of the internal bipolaron state (see text) at $a_{I I} / \xi=0.6$ are indicated with the dotted lines. Also, the upper bound for the ground-state energy of two separate polarons $\left(E_{2 \mathrm{pol}}\right)$ and the corresponding effective mass $\left(m_{2 \mathrm{pol}}^{*}\right)$ are shown.

a function of the coupling parameter $\alpha$ at $a_{I I}=10$. For $\alpha<$ 2.71 the system consists of two separate polarons, while for $\alpha>2.71$ a bipolaron is formed. For two separate polarons the bipolaron radius is defined as infinity. As a function of $\alpha$ the effective mass exhibits an increasing behavior, and for $\alpha>3$ it increases more rapidly, indicating the transition to the strong-coupling regime, which is also present for a single polaron [11]. If the bipolaron is stable, the bipolaron radius decreases as a function of $\alpha$. This shows that the bipolaron becomes more tightly bound as the coupling is increased.

In Fig. 5 the upper bound (31), the polaronic effective mass (33), and the inverse bipolaron radius (23) are presented as a function of the impurity-impurity scattering length $a_{I I}$ at $\alpha=2$ (region 1 in Fig. 3). The energy and the effective mass of two polarons are also shown. This reveals that the bipolaron is only stable for sufficiently small values of $a_{I I}$, with a polaronbipolaron transition at $a_{I I} / \xi=1.3$. From Fig. 5 it is clear that in this case the polaron-bipolaron transition is accompanied with a discontinuity in the effective mass. The value $a_{I I} / \xi=$ 0.6 is also indicated in Fig. 5, which corresponds to another discontinuity in the effective mass, as well as in the bipolaron radius. For $a_{I I} / \xi<0.6$ the bipolaron is relatively small and heavy, and the ground-state energy and the properties exhibit a strong dependence on $a_{I I}$ compared to the behavior for $a_{I I} / \xi>0.6$. This suggests that for $a_{I I} / \xi<0.6$ the bipolaron can be considered as a tightly bound particle, while for $a_{I I} / \xi>$ 0.6 it consists of a more loosely bound state of two polarons. Increasing $a_{I I}$ results in a less tightly bound bipolaron and finally in the formation of two separate polarons.

In Fig. 6 the upper bound (31), the polaronic effective mass (33), and the inverse bipolaron radius (23) are presented as a function of the impurity-impurity scattering length $a_{I I}$ at $\alpha=2.85$ (region 2 in Fig. 3). The energy of two separate
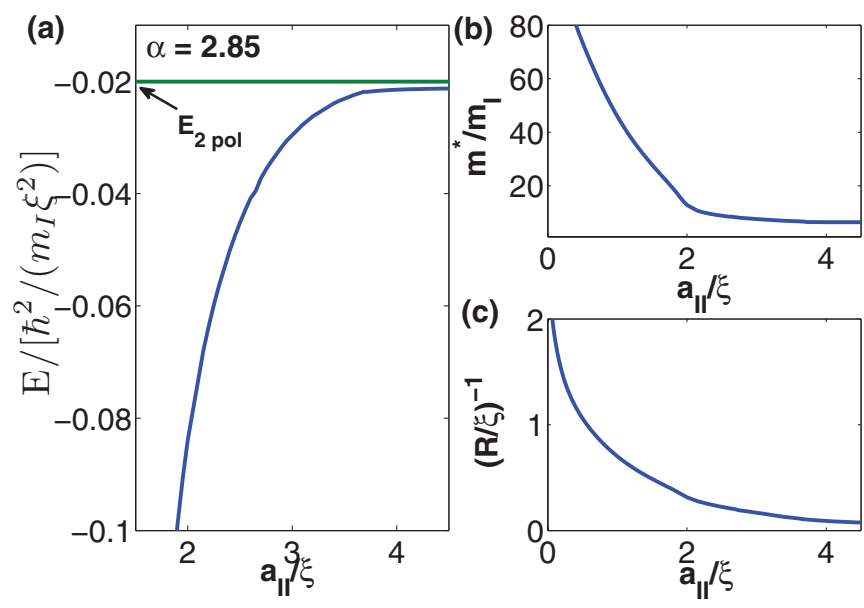

FIG. 6. (Color online) (a) The upper bound of the ground-state energy, (b) the effective mass, and (c) the inverse of the bipolaron radius are presented as a function of the impurity-impurity scattering length $a_{I I}$ at $\alpha=2.85$ (region 2 in Fig. 3) for two ${ }^{6} \mathrm{Li}$ impurities in a $\mathrm{Na}$ condensate. The upper bound for the ground-state energy of two polarons $\left(E_{2 \mathrm{pol}}\right)$ is also indicated.

polarons is also indicated, which shows that the bipolaron is always stable, irrespective of $a_{I I}$. Also, here we find that increasing $a_{I I}$ results in a less tightly bound bipolaron. Again, we can distinguish two regimes in the $a_{I I}$ dependence of $E$ and $m^{*}$ but now without a discontinuity at the transition. At small $a_{I I}$ the increase of the ground-state energy and the decrease of the effective mass as a function of $a_{I I}$ are significantly faster than at higher values of $a_{I I}$, with a transition region at $a_{I I} / \xi \approx 2$. As before, this indicates that at small values of $a_{I I}$ the bipolaron can be considered a tightly bound particle, while at large values it is more appropriately interpreted as two loosely bound polarons. Moreover, at small values for $a_{I I}$ the variationally determined vector $\vec{a}$, separating the two impurities in the trial system of Fig. 1, is zero, while for relatively large $a_{I I}$ it is finite. This shows that the loosely bound polarons at large $a_{I I}$ are spatially separated, and the shape can be interpreted as a dumbbell.

Finally, in Fig. 7 the upper bound (31), the polaronic effective mass (33), and the inverse bipolaron radius (23) are presented as a function of the impurity-impurity scattering length $a_{I I}$ at $\alpha=4$ (region 3 in Fig. 3). The upper bound (31) in the limit $a_{I I} \rightarrow \infty$ is also indicated, which is lower than the upper bound of the ground-state energy for two separate polarons. This shows that the bipolaron is always stable. Also, here we observe two regimes as a function of $a_{I I}$ with a transition at $a_{I I} / \xi=0.91$ which is accompanied by a discontinuity in the effective mass and the bipolaron radius, indicating a transition between a tightly bound bipolaron and a more loosely bound state of two polarons. Also, in this case the variationally determined vector $\vec{a}$ is zero for the tightly bound bipolaron and nonzero for the loosely bound state of two polarons, resulting in a dumbbell bipolaron.

\section{STRONG-COUPLING FORMALISM}

We now apply a generalization of the Landau-Pekar strongcoupling treatment for $N_{I}$ polaronic particles to impurities 

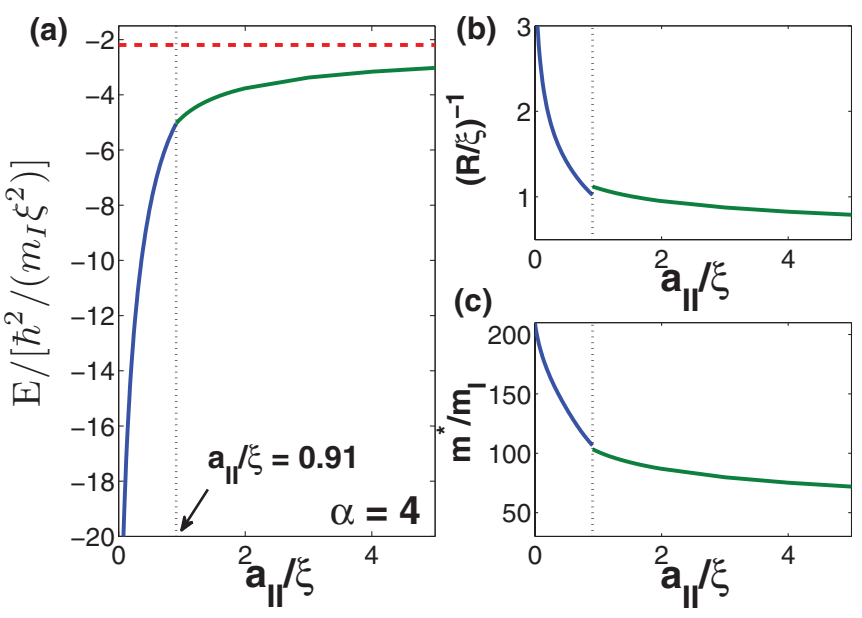

FIG. 7. (Color online) (a) The upper bound of the ground-state energy, (b) the inverse of the bipolaron radius, and (c) the effective mass are presented as a function of the impurity-impurity scattering length $a_{I I}$ at $\alpha=4$ (region 3 in Fig. 3) for two ${ }^{6} \mathrm{Li}$ impurities in a $\mathrm{Na}$ condensate. The dotted line at $a_{I I}=0.91 \xi$ indicates a transition of the internal state of the bipolaron (see text). The dashed line shows the upper bound to the ground-state energy in the limit $a_{I I} \rightarrow \infty$.

in a Bose-Einstein condensate to examine the formation of multipolarons.

\section{A. Formalism}

For the description of the strong-coupling formalism the product ansatz is used, which states that the total wave function $|\Phi\rangle$ is the product of a part that describes the Bogoliubov excitations $|\phi\rangle$ and a part for the impurities $\left|\Psi^{\left(N_{I}\right)}\right\rangle:|\Phi\rangle=$ $|\phi\rangle\left|\Psi^{\left(N_{I}\right)}\right\rangle$. Taking the expectation value of the Fröhlich Hamiltonian (5) with respect to $|\Phi\rangle$ and completing the squares for the Bogoliubov creation and annihilation operators result in

$$
\begin{aligned}
\left\langle\Phi\left|\hat{H}_{\mathrm{pol}}^{\left(N_{I}\right)}\right| \Phi\right\rangle= & K+\sum_{\vec{k} \neq 0} \hbar \omega_{\vec{k}}\langle\phi|\left(\hat{\alpha}_{\vec{k}}^{\dagger}+\frac{V_{\vec{k}} \rho_{I}(\vec{k})}{\hbar \omega_{\vec{k}}}\right) \\
& \times\left(\hat{\alpha}_{\vec{k}}+\frac{V_{\vec{k}}^{*} \rho_{I}^{\dagger}(\vec{k})}{\hbar \omega_{\vec{k}}}\right)|\phi\rangle-\sum_{\vec{k} \neq 0} \frac{\left|V_{\vec{k}} \hat{\rho}_{I}(\vec{k})\right|^{2}}{\hbar \omega_{\vec{k}}}+U,
\end{aligned}
$$

where $K$ is the kinetic energy, $\rho_{I}(\vec{k})$ is the Fourier transform of the density, and $U$ is the mutual interaction energy of the impurities:

$$
\begin{gathered}
K=\left\langle\Psi^{\left(N_{I}\right)}\left|\sum_{i=1}^{N_{I}} \frac{\hat{p}_{i}^{2}}{2 m_{I}}\right| \Psi^{\left(N_{I}\right)}\right\rangle, \\
\rho_{I}(\vec{k})=\left\langle\Psi^{\left(N_{I}\right)}\left|\sum_{i=1}^{N_{I}} e^{i \vec{k} \cdot \hat{\vec{r}}_{i}}\right| \Psi^{\left(N_{I}\right)}\right\rangle, \\
U=\left\langle\Psi^{\left(N_{I}\right)}\left|\sum_{i<j}^{N_{I}} V_{I I}\left(\hat{\vec{r}}_{i}-\hat{\vec{r}}_{j}\right)\right| \Psi^{\left(N_{I}\right)}\right\rangle .
\end{gathered}
$$

The expectation value of Hamiltonian (34) is minimal if the wave function of the Bogoliubov excitations is chosen as the vacuum $\left|\phi_{g}\right\rangle$ for the "displaced" operators:

$$
\left\langle\phi_{g}\left|\left(\hat{\alpha}_{\vec{k}}^{\dagger}+\frac{V_{\vec{k}} \rho_{I}(\vec{k})}{\hbar \omega_{\vec{k}}}\right)\left(\hat{\alpha}_{\vec{k}}+\frac{V_{\vec{k}}^{*} \rho_{I}^{\dagger}(\vec{k})}{\hbar \omega_{\vec{k}}}\right)\right| \phi_{g}\right\rangle=0 .
$$

This results in the following expression for the ground-state energy:

$$
E_{0}^{\left(N_{I}\right)}=K-\sum_{\vec{k} \neq 0} \frac{\left|V_{\vec{k}}\right|^{2}\left|\rho_{I}(\vec{k})\right|^{2}}{\hbar \omega_{\vec{k}}}+U
$$

This result can alternatively be derived with a canonical transformation, as done by Bogoliubov and Tyablikov for a single polaron [50]. For the impurities we will use a variational wave function; the resulting energy (39) is then an upper bound for the ground-state energy.

\section{Effective mass}

The strong-coupling formalism allows a derivation of the multipolaron effective mass in a similar way as was done for a single polaron in Refs. [51-54] and for the bipolaron in Ref. [55]. The total momentum of the polaronic system $\hat{\overrightarrow{\mathcal{P}}}$ is given by

$$
\hat{\overrightarrow{\mathcal{P}}}=\hat{\vec{P}}+\sum_{\vec{k}} \hbar \vec{k} \hat{\alpha}_{\vec{k}}^{\dagger} \hat{\alpha}_{\vec{k}}
$$

with $\hat{\vec{P}}=\sum_{i}^{N_{I}} \hat{\vec{p}}_{i}$. This operator commutes with the Fröhlich Hamiltonian (5), and the total momentum is thus a constant of motion: $\langle\hat{\mathcal{P}}\rangle=\overrightarrow{\mathcal{P}}$. We make this explicit by means of a Lagrange multiplier $\vec{v}$ which physically represents the velocity of the system and consider the operator

$$
\hat{H}_{\mathrm{pol}}^{\left(N_{I}\right)}(\vec{v})=\hat{H}_{\mathrm{pol}}^{\left(N_{I}\right)}-\vec{v} \cdot\left(\hat{\vec{P}}+\sum_{\vec{k}} \hbar \vec{k} \hat{\alpha}_{\vec{k}}^{\dagger} \hat{\alpha}_{\vec{k}}-\overrightarrow{\mathcal{P}}\right)
$$

for minimization. The effective mass $m^{*}$ of the multipolaron can then be determined from the relation $\overrightarrow{\mathcal{P}}=m^{*} \vec{v}$. The impurity variational wave function $\left|\Psi^{\left(N_{I}\right)}\right\rangle$, with $\langle\hat{\vec{P}}\rangle=0$, has to be adapted to a wave function with finite averaged momentum $\langle\hat{\vec{P}}\rangle=N_{I} m_{I} \vec{v}$; we use

$$
\Psi^{\prime\left(N_{I}\right)}\left(\left\{\vec{r}_{i}\right\}\right)=\exp \left[\frac{i m_{I} \vec{v} \cdot \sum \vec{r}_{i}}{\hbar}\right] \Psi^{\left(N_{I}\right)}\left(\left\{\vec{r}_{i}\right\}\right) .
$$

Taking the expectation value of $\hat{H}_{\text {pol }}^{\left(N_{I}\right)}(\vec{v})$ with respect to the product wave function $|\phi\rangle\left|\Psi^{\prime\left(N_{I}\right)}\right\rangle$ and introducing the wave function $\left|\phi_{g}\right\rangle$ for the Bogoliubov excitations, as in (38), result in

$$
E^{\left(N_{I}\right)}(\vec{v})=K+U-N_{I} \frac{m_{I} v^{2}}{2}+v \cdot \overrightarrow{\mathcal{P}}-\sum_{\vec{k} \neq 0} \frac{\left|V_{\vec{k}}\right|^{2}\left|\rho_{I}(\vec{k})\right|^{2}}{\hbar \omega_{\vec{k}}-\hbar \vec{v} \cdot \vec{k}},
$$

with $K, \rho_{I}(\vec{k})$, and $U$ as defined in (35), (36), and (37), respectively. Minimizing expression (43) with respect to $\vec{v}$ and performing a Taylor expansion for small $\vec{v}$ gives, for the 
effective mass,

$$
m^{*}=N_{I} m_{I}+2 \hbar^{2} \sum_{\vec{k} \neq 0} \frac{\left|V_{\vec{k}}\right|^{2}\left|\rho_{I}(\vec{k})\right|^{2}}{\left(\hbar \omega_{\vec{k}}\right)^{3}} k_{z}^{2},
$$

with $k_{z}$ being the $z$ component of $\vec{k}$.

\section{Variational impurity wave function}

For $N_{I}$ distinguishable particles we consider the following normalized variational wave function:

$$
\Psi^{\left(N_{I}\right)}\left(\left\{\vec{r}_{i}\right\}\right)=\prod_{i=1}^{N_{I}} \frac{1}{\left(\pi \lambda^{2}\right)^{3 / 4}} \exp \left[-\frac{\left(\vec{r}_{i}-\vec{a}_{i}\right)^{2}}{2 \lambda^{2}}\right],
$$

which consists of $N_{I}$ Gaussians with standard deviation $\lambda$, centered at $\vec{a}_{i}$. For a single impurity in a condensate a numerical calculation of the wave function revealed a good agreement with a Gaussian wave function if the polaronic coupling is strong enough [56]. The corresponding expectation values are

$$
\begin{gathered}
K=N_{I} \frac{3 \hbar^{2}}{4 m_{I} \lambda^{2}}, \\
U=\sum_{i<j}^{N_{I}} \sum_{\vec{k}} V_{I I}(\vec{k}) e^{-\frac{k^{2} \lambda^{2}}{2}+i \vec{k} \cdot\left(\vec{a}_{1}-\vec{a}_{2}\right)}, \\
\rho_{I}(\vec{k})=\exp \left[-\frac{k^{2} \lambda^{2}}{4}\right] \sum_{i=1}^{N_{I}} e^{i \vec{k} \cdot \vec{a}_{i}} .
\end{gathered}
$$

Wave function (45) can be extended to the case of identical impurities by using a Slater determinant for fermions or the appropriate symmetrized wave function for bosons.

\section{Single-polaron limit}

If all the impurities are infinitely separated, we expect the multipolaron to reduce to individual polarons. For the impurity wave function (45) this corresponds to the limit $\left|\vec{a}_{i}-\vec{a}_{j}\right| \rightarrow$ $\infty \forall i \neq j$. The upper bound for the polaron ground-state energy (39) becomes in this limit

$$
\begin{aligned}
\lim _{\substack{\left|\vec{a}_{i}-\vec{a}_{j}\right| \rightarrow \infty \\
\forall i \neq j}} E_{0}^{\left(N_{I}\right)} & =N_{I}\left(\frac{3 \hbar^{2}}{4 m_{I} \lambda^{2}}-\sum_{\vec{k} \neq 0} \frac{\left|V_{\vec{k}}\right|^{2}}{\varepsilon_{\vec{k}}} e^{-\frac{k^{2} \lambda^{2}}{2}}\right) \\
& =N_{I} E_{0}^{(1)} .
\end{aligned}
$$

This equals $N_{I}$ times the strong-coupling result for the upper bound for the ground-state energy of a single polaron $E_{0}^{(1)}$, as expected. The effective mass of the multipolaron (44) becomes in this limit

$$
\begin{aligned}
\lim _{\substack{\left|\vec{a}_{i}-\vec{a}_{j}\right| \rightarrow \infty \\
\forall i \neq j}} m^{*} & =N_{I}\left(m_{I}+2 \hbar^{2} \sum_{\vec{k} \neq 0} \frac{\left|V_{\vec{k}}\right|^{2}}{\left(\hbar \omega_{\vec{k}}\right)^{3}} e^{-\frac{k^{2} \lambda^{2}}{2}} k_{z}^{2}\right) \\
& =N_{I} m_{\mathrm{pol}}^{*} .
\end{aligned}
$$

This is $N_{I}$ times the strong-coupling result for the effective mass of a single polaron $m_{\mathrm{pol}}^{*}$, again as expected.

\section{Impurities in a Bose-Einstein condensate}

We now consider the specific polaronic system consisting of impurities in a BEC. Introducing the Bogoliubov dispersion
(6), the interaction amplitude (7), and the variational wave function (45) for $N_{I}$ distinguishable impurities in the upper bound for the ground-state energy (39) gives, in polaronic units $\left(\hbar=m_{I}=\xi=1\right)$,

$$
\begin{aligned}
E_{0}^{\left(N_{I}\right)}= & \frac{3 N_{I}}{4 \lambda^{2}}+\frac{2 a_{I I}}{(2 \pi)^{1 / 2} \lambda^{3}} \sum_{i<j}^{N_{I}} \exp \left[-\frac{\left(\vec{a}_{i}-\vec{a}_{j}\right)^{2}}{2 \lambda^{2}}\right] \\
& -\frac{2 \alpha \mu}{\pi} \int_{0}^{\infty} d k k^{2} \frac{\exp \left[-\frac{k^{2} \lambda^{2}}{2}\right]}{k^{2}+2} \sum_{i, j}^{N_{I}} \frac{\sin \left[k\left|\vec{a}_{i}-\vec{a}_{j}\right|\right]}{k\left|\vec{a}_{i}-\vec{a}_{j}\right|},
\end{aligned}
$$

where a contact pseudopotential with the first-order Lippmann-Schwinger result was used for the impurityimpurity interaction. We also introduced expression (32) for the dimensionless polaronic coupling parameter $\alpha$ and the dimensionless mass factor,

$$
\mu=\frac{\left(m_{B}+m_{I}\right)^{2}}{4 m_{B} m_{I}} .
$$

In the strong-coupling regime the mass parameter $m_{B} / m_{I}$ and the coupling parameter $\alpha$ combine to a single dimensionless coupling parameter $\alpha \mu$. The effective mass of $N_{I}$ impurities in a BEC in polaronic units can be written as

$$
m^{*}=N_{I} m_{I}+\frac{4 \alpha \mu m_{B}^{2}}{\pi^{2}} \int d \vec{k} \frac{\left|\rho_{I}(\vec{k})\right|^{2}}{\left(2+k^{2}\right)^{2}} \frac{k_{z}^{2}}{k^{2}} .
$$

\section{B. Results}

\section{Bipolaron}

First, we examine two impurities in a Bose-Einstein condensate $\left(N_{I}=2\right)$ and the formation of a bipolaron. The bipolaron radius, estimated by the mean-square distance between the impurities (23), gives, for the impurity variational wave function (45),

$$
R=\sqrt{\left\langle\left(\vec{r}_{1}-\vec{r}_{2}\right)^{2}\right\rangle}=\sqrt{a^{2}+3 \lambda^{2}},
$$

with $a=\left|\vec{a}_{1}-\vec{a}_{2}\right|$.

In Fig. 8 the $\left(a_{I I}, \alpha \mu\right)$-phase diagram of two distinguishable impurity atoms in a BEC is presented, and the different regions as a function of $\alpha$ are also indicated. With each $a_{I I}$ a value $(\alpha \mu)_{\text {exist }}$ is associated in such a way that for $\alpha \mu<(\alpha \mu)_{\text {exist }}$ the minimization of the right-hand side of expression (51) yields no solution at a finite value of $\lambda$. If $\alpha \mu>(\alpha \mu)_{\text {exist }}$, another value $(\alpha \mu)_{\text {stable }}$ can be determined, separating a region with a metastable bipolaron (positive ground-state energy) for $\alpha \mu<(\alpha \mu)_{\text {stable }}$ from a region with a stable bipolaron (negative ground-state energy) for $\alpha \mu>(\alpha \mu)_{\text {stable. }}$. In the case of a metastable bipolaron the impurities are expected to be expelled from the condensate. However, since the formalism is only expected to be valid at strong coupling, the physical relevance of the metastable bipolaron is not obvious. In the limit $a_{I I} \rightarrow \infty$ the strong-coupling results for a single polaron are retrieved: $(\alpha \mu)_{\text {exist }}=3.57$ and $(\alpha \mu)_{\text {stable }}=3.84$ [54]. For $\alpha \mu>3.84$ (region 3) the formation of the bipolaron is always energetically favorable compared to the formation of two separate polarons, irrespective of $a_{I I}$. For $\alpha \mu \in[3.39,3.84]$ (region 2) a stable solution only exists if $a_{I I}$ is smaller than 


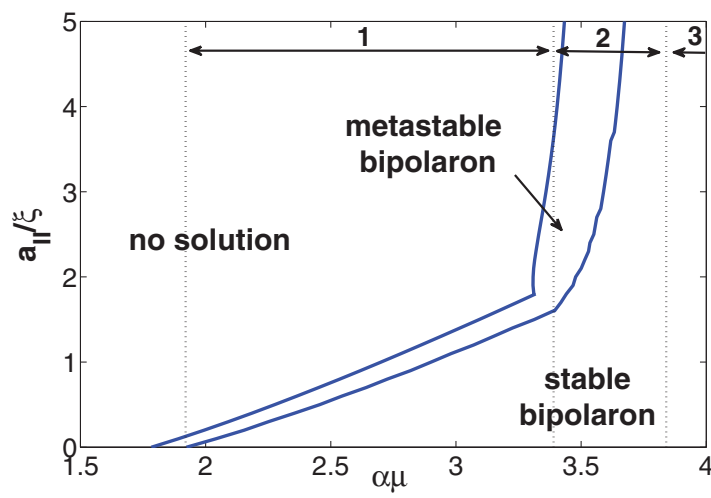

FIG. 8. (Color online) The solid lines show the $\left(a_{I I}, \alpha \mu\right)$-phase diagram for two distinguishable impurity atoms in a Bose-Einstein condensate with $\alpha \mu$ being the coupling parameter and $a_{I I}$ being the impurity-impurity scattering length as calculated with the strongcoupling formalism. At strong coupling the bipolaron is stable, and at sufficiently weak coupling there is no solution. In between there is a region where the solution results in a positive ground-state energy, which means the bipolaron is metastable. The dotted lines at $\alpha \mu=1.92, \alpha \mu=3.39$, and $\alpha \mu=3.84$ indicate the boundaries of the different regions, as discussed in the text.

a critical value, which is relatively large and increases rapidly as a function of $\alpha \mu$. For $\alpha \mu \in[1.92,3.39]$ (region 1) a stable bipolaron also only exists for $a_{I I}$ smaller than a critical value, but now this critical value is relatively low and increases much more slowly as a function of $\alpha \mu$, compared to the behavior in region 2 . At weaker coupling there is never a stable solution. Considering the stable solution, the variationally determined vector $\vec{a}_{1}-\vec{a}_{2}$, which represents the separation between the two Gaussians in wave function (45), is always zero if $\alpha \mu<3.39$; for $\alpha \mu>3.39$ it is finite at sufficiently large values of $a_{I I}$, resulting in a dumbbell bipolaron.

In Fig. 9 the upper bound for the ground-state energy (51), the inverse bipolaron radius (54), and the effective mass (53)
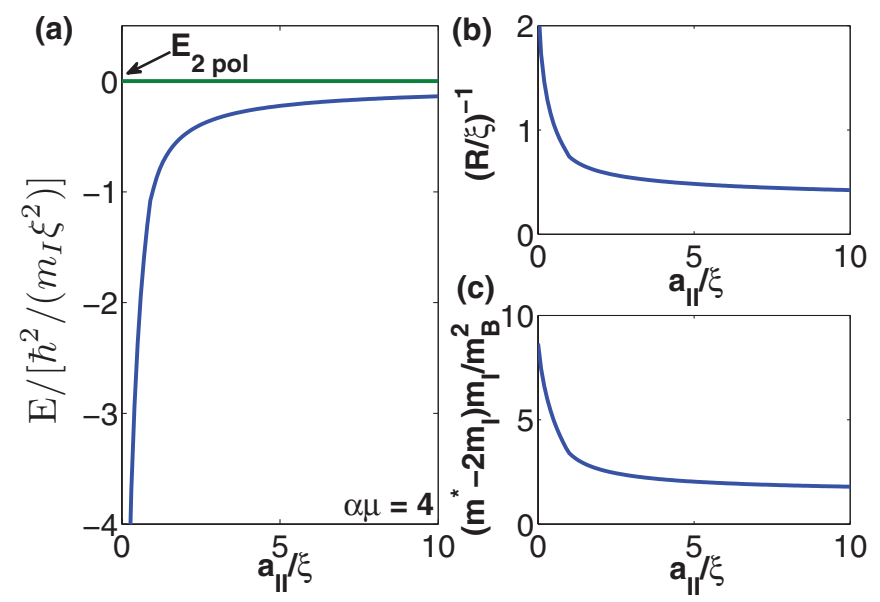

FIG. 9. (Color online) (a) The upper bound for the ground-state energy, (b) the inverse bipolaron radius, and (c) the effective mass as a function of the impurity-impurity scattering length $a_{I I}$ at $\alpha \mu=4$ for two distinguishable impurities in a Bose-Einstein condensate. The upper bound for the ground-state energy of two polarons $E_{2 \text { pol }}$ is also indicated. are presented as a function of the impurity-impurity scattering length $a_{I I}$ for two distinguishable impurities in a BEC at $\alpha \mu=$ 4. The upper bound for the ground-state energy of a single polaron is also indicated, which shows that the formation of a bipolaron is energetically favorable for all finite values of $a_{I I}$. From Fig. 9 we see that for increasing $a_{I I}$ the bipolaron binding energy decreases, the radius increases, and the effective mass decreases, showing that the bipolaron becomes less tightly bound. In the limit $a_{I I} \rightarrow \infty$ the bipolaron effective mass becomes twice the effective mass of a single polaron. Considering the $a_{I I}$ dependence of the properties reveals two regimes with a transition at $a_{I I} / \xi=0.85$. For $a_{I I} / \xi<0.85$ there is a relatively strong dependence of the properties on $a_{I I}$ compared to the behavior for $a_{I I} / \xi>0.85$. Furthermore, the variationally determined vector $\vec{a}_{1}-\vec{a}_{2}$, representing the distance between the two centers of the Gaussians in wave function (45), is only nonzero for $a_{I I} / \xi>0.85$. This is consistent with our earlier interpretations in that for $a_{I I} / \xi<$ 0.85 the bipolaron is tightly bound, while for $a_{I I} / \xi>0.85$ it is a more loosely bound dumbbell bipolaron.

A similar analysis can be made for identical impurities by antisymmetrizing wave function (45) for fermions or symmetrizing it for bosons. In the case of two identical bosons the same qualitative results are retrieved as for distinguishable impurities. For identical fermions the symmetry of the wave function results in a vanishing expectation value of the $s$-wave contact pseudopotential (47), which implies that at ultralow temperatures identical fermions behave as noninteracting particles. The only remaining parameter is the coupling parameter $\alpha \mu$, and we find $(\alpha \mu)_{\text {exist }}=3.09$ as a minimum for a solution to exist and $(\alpha \mu)_{\text {stable }}=3.31$ as a minimum to find a stable solution. Furthermore, if a solution exists, it is always energetically favorable to form a bipolaron compared to two separate polarons.

\section{Multipolaron}

We now examine the multipolaron by minimizing the upper bound for the ground-state energy (51) for $N_{I}=1,2, \ldots, 8$ distinguishable impurities. In Ref. [30] a similar procedure was presented, but the expectation values of the positions of the impurities were considered to be equal, which corresponds to the variational wave function (45) with $\vec{a}_{i}=\vec{a}_{j} \forall i \neq j$.

In Fig. 10 (a) the resulting phase diagram is presented for $N_{I}=1,2, \ldots, 8$ distinguishable impurities in a Bose-Einstein condensate as a function of the polaronic coupling $\alpha \mu$ and the impurity-impurity scattering length $a_{I I}$. Similar to before for the bipolaron for each $a_{I I}$ a minimum coupling value $(\alpha \mu)_{\text {exist }}$ is needed for a solution of the minimization of (51) to exist at finite $\lambda$, and another minimum value $(\alpha \mu)_{\text {stable }}$ $\left[(\alpha \mu)_{\text {stable }}>(\alpha \mu)_{\text {exist }}\right]$ is necessary to find a stable solution. In the limit $a_{I I} \rightarrow \infty$ the one-polaron strong-coupling results are found for any number of impurities: $(\alpha \mu)_{\text {exist }}=3.57$ and $(\alpha \mu)_{\text {stable }}=3.84$. In Fig. 10(a) only $(\alpha \mu)_{\text {stable }}$ is presented for clarity. This shows that if the number of impurities $N_{I}$ is increased, $(\alpha \mu)_{\text {stable }}$ decreases, resulting in a larger stability region. This behavior of a smaller critical coupling value for the formation of a larger multipolaron was also observed in Ref. [30]. 
(a)

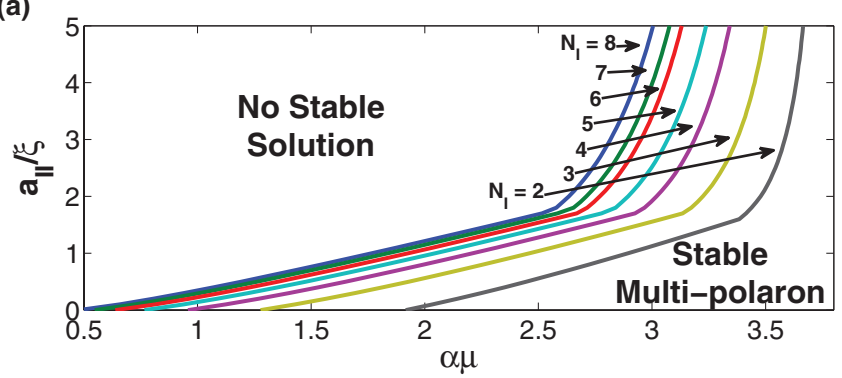

(b)

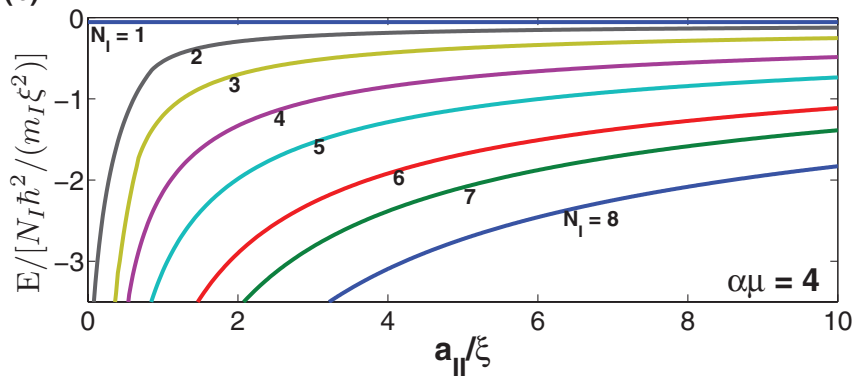

FIG. 10. (Color online) In (a) the $\left(a_{I I}, \alpha \mu\right)$-phase diagram for $N_{I}$ distinguishable impurities in a Bose-Einstein condensate with respect to the formation of a stable multipolaron is presented, with $\alpha \mu$ being the coupling parameter and $a_{I I}$ being the impurity-impurity scattering length. In (b) the upper bound for the multipolaron ground-state energy per impurity is presented as a function of the impurity-impurity scattering length $a_{I I}$ for distinguishable impurities at $\alpha \mu=4$.

The $\left(a_{I I}, \alpha \mu\right)$-phase diagram in Fig. 10(a) for a specific number $N_{I}$ of impurities is qualitatively the same as for the bipolaron in Fig. 8. This means the same qualitative regions can be distinguished as a function of $\alpha \mu$ as we did for the bipolaron in Fig. 8, and we present a general analysis, valid for every value of $N_{I}$. In region 3 the formation of the multipolaron is always energetically favored compared to $N_{I}$ separate polarons. Furthermore, the variationally determined locations of the impurities $\vec{a}_{i}$ coincide at sufficiently small $a_{I I}$, indicating a tightly bound multipolaron, while at sufficiently large $a_{I I}$ we find $\vec{a}_{i} \neq \vec{a}_{j} \forall i \neq j$, indicating a bound droplet of $N_{I}$ separate polarons (similar to the dumbbell bipolaron). In region 2 the minimization of the right-hand side of (51) yields no stable solution if $a_{I I}$ is larger than a critical value, which is relatively large and increases rapidly as a function of $\alpha \mu$. In region 1 there is also no stable solution for $a_{I I}$ above a critical value, which is now relatively low and increases slowly as a function of $\alpha \mu$. In this regime the behavior of the critical $a_{I I}$ as a function of $\alpha \mu$ resembles a straight line, and it was shown in Ref. [30] that in the limit $N_{I} \rightarrow \infty$ this line is well approximated by the boundary for phase separation [57-60]:

$$
\frac{a_{I I}}{\xi}=\alpha \mu
$$

At small $\alpha \mu$ there is never a solution.

In Fig. 10(b) the upper bound for the multipolaron groundstate energy per impurity is presented as a function of the impurity-impurity scattering length $a_{I I}$ at $\alpha \mu=4$. For $a_{I I} \rightarrow \infty$ all curves tend to the single-polaron result. At finite $a_{I I}$ the ground-state energy per particle decreases as $N_{I}$ is increased, showing that it is energetically favorable for the impurities to cluster and form a multipolaron.

For the positive impurity-boson scattering length there is a depletion of the condensate in the vicinity of the multipolaron. This can be detrimental for the polaronic description since the Bogoliubov approximation breaks down at large depletion. This restriction is only important for the tightly bound multipolaron at small $a_{I I}$. In the case of a loosely bound droplet of separate polarons at sufficiently large $a_{I I}$ the mean distance between the impurities is of the order of the healing length, which shows that the depletion is spread out over a large volume and the Bogoliubov approximation is not jeopardized. For the tightly bound multipolaron, on the other hand, these considerations result in a critical number of impurities above which the system cannot be described by the Fröhlich Hamiltonian. It was shown in Ref. [30] that for typical experimental parameters only tightly bound multipolarons with a few impurities are possible.

\section{COMPARISON OF THE BIPOLARON RESULTS FROM THE TWO FORMALISMS}

The results of the strong-coupling formalism of Sec. IV A, applied to the specific system of two distinguishable ${ }^{6} \mathrm{Li}$ impurities in a Na condensate $\left(m_{B} / m_{I}=3.82\right.$ and $\left.\mu=1.52\right)$, can be compared with the results of the path-integral treatment of Sec. III. The phase diagrams in Figs. 3 and 8 exhibit a similar qualitative behavior, and we can compare the three regions denoted in the figures separately. Both formalisms predict that there is no formation of a bipolaron if the polaronic coupling is too weak, and at slightly stronger coupling (region 1) the bipolaron is only formed at relatively small $a_{I I}$. In region 2 the all-coupling approach predicts the bipolaron is always formed, while according to the strong-coupling approach there is no bipolaron formation at very high values of $a_{I I}$. In region 3 both formalisms agree on the prediction that the bipolaron is always formed, irrespective of $a_{I I}$. Quantitatively, the strong-coupling formalism underestimates the critical coupling parameter for bipolaron formation, compared to the all-coupling approach. Note that the strong-coupling approach also underestimates the critical coupling value for the transition to the strong-coupling regime, compared to a numerical study [56].

Considering the $a_{I I}$ dependence of the properties, both formalisms reveal two distinct regimes. The behavior at relatively small $a_{I I}$ corresponds to a tightly bound bipolaron that behaves as a single particle, while at relatively large $a_{I I}$ it is better interpreted as a loosely bound dumbbell bipolaron. The all-coupling approach predicts a possible discontinuity in the polaronic properties at this transition which diminishes as the coupling is increased and ultimately, well in the strong-coupling regime, vanishes, as also predicted by the strong-coupling treatment.

\section{CONCLUSIONS}

The Feynman all-coupling polaron treatment was applied for two distinguishable impurities in a condensate. This showed that if the polaronic coupling is strong enough, a bipolaron is formed. We also calculated the bipolaron effective mass and the bipolaron radius. Considering the 
dependence of the polaronic properties on the impurityimpurity scattering length, $a_{I I}$ results in the distinction of two regimes as a function of $a_{I I}$, a tightly bound bipolaron that behaves as a single particle at relatively small $a_{I I}$ and a more loosely bound dumbbell bipolaron at sufficiently large $a_{I I}$. If the coupling is sufficiently strong or weak, this transition is found to be accompanied by a discontinuity in the properties of the bipolaron, which becomes less pronounced as the coupling is increased towards the strong-coupling regime.

We also applied a strong-coupling treatment to impurities in a Bose-Einstein condensate. For two distinguishable impurities in a BEC this leads to similar results, as found by the all-coupling treatment. This strong-coupling treatment was then extended for identical impurities. For identical bosons this results in the same qualitative results as for distinguishable impurities. For identical fermions the mutual $s$-wave interaction vanishes, and above a critical coupling strength the formation of a bipolaron is always energetically favored compared to two separate polarons.

The strong-coupling treatment was then applied for more impurities in a BEC to consider the formation of multipolarons. We find that the multipolaron becomes stable at weaker coupling as the number of impurities is increased. Furthermore, the ground-state energy per particle decreases as $N_{I}$ is increased, which shows that clustering is energetically favorable in the strong-coupling regime.

Since both formalisms are variational and depend on the choice of a model system, there is no guarantee that they describe the actual system. However, the extension of the allcoupling Feynman approach for the bipolaron not only uses the model system to describe the system but also incorporates corrections and is expected to be more accurate compared to the usual variational principle. Furthermore, the agreement between the two formalisms at strong coupling indicates that the physics of the system is captured by the models.
[1] I. Bloch, J. Dalibard, and W. Zwerger, Rev. Mod. Phys. 80, 885 (2008).

[2] D. K. K. Lee and J. M. F. Gunn, Phys. Rev. B 46, 301 (1992).

[3] F. M. Cucchietti and E. Timmermans, Phys. Rev. Lett. 96, 210401 (2006)

[4] K. Sacha and E. Timmermans, Phys. Rev. A 73, 063604 (2006).

[5] A. S. Alexandrov and J. T. Devreese, Advances in Polaron Physics (Springer, Heidelberg, 2009).

[6] J. T. Devreese, arXiv:1012.4576.

[7] R. P. Feynman, Phys. Rev. 97, 660 (1955).

[8] H. Fröhlich, Adv. Phys. 3, 325 (1954).

[9] L. D. Landau and S. I. Pekar, Zh. Eksp. Teor. Fiz. 16, 341 (1946).

[10] L. D. Landau and S. I. Pekar, Zh. Eksp. Teor. Fiz. 18, 419 (1948).

[11] J. Tempere, W. Casteels, M. K. Oberthaler, S. Knoop, E. Timmermans, and J. T. Devreese, Phys. Rev. B 80, 184504 (2009).

[12] A. Novikov and M. Ovchinnikov, J. Phys. B 43, 105301 (2010).

[13] N. Spethmann, F. Kindermann, S. John, C. Weber, D. Meschede, and A. Widera, Phys. Rev. Lett. 109, 235301 (2012).

[14] A. Härter, A. Krükow, A. Brunner, W. Schnitzler, S. Schmid, and J. H. Denschlag, Phys. Rev. Lett. 109, 123201 (2012).

[15] L. Ratschbacher, C. Zipkes, C. Sias, and M. Kohl, Nat. Phys. 8, 649 (2012).

[16] N. Spethmann, F. Kindermann, S. John, C. Weber, D. Meschede, and A. Widera, Appl. Phys. B 106, 513 (2012).

[17] J. Catani, G. Lamporesi, D. Naik, M. Gring, M. Inguscio, F. Minardi, A. Kantian, and T. Giamarchi, Phys. Rev. A 85, 023623 (2012).

[18] T. Takekoshi, M. Debatin, R. Rameshan, F. Ferlaino, R. Grimm, H.-C. Nägerl, C. R. Le Sueur, J. M. Hutson, P. S. Julienne, S. Kotochigova, and E. Tiemann, Phys. Rev. A 85, 032506 (2012).

[19] J. W. Park, C.-H. Wu, I. Santiago, T. G. Tiecke, S. Will, P. Ahmadi, and M. W. Zwierlein, Phys. Rev. A 85, 051602 (2012).

[20] T. Schuster, R. Scelle, A. Trautmann, S. Knoop, M. K. Oberthaler, M. M. Haverhals, M. R. Goosen, S. J. J. M. F. Kokkelmans, and E. Tiemann, Phys. Rev. A 85, 042721 (2012).
[21] M. Repp, R. Pires, J. Ulmanis, R. Heck, E. D. Kuhnle, M. Weidemüller, and E. Tiemann, Phys. Rev. A 87, 010701 (2013).

[22] W. Casteels, J. Tempere, and J. T. Devreese, Phys. Rev. A 83, 033631 (2011).

[23] A. S. Mishchenko, N. Nagaosa, N. V. Prokof'ev, A. Sakamoto, and B. V. Svistunov, Phys. Rev. Lett. 91, 236401 (2003).

[24] J. Devreese, J. De Sitter, and M. Goovaerts, Phys. Rev. B 5, 2367 (1972).

[25] L. Viverit, C. J. Pethick, and H. Smith, Phys. Rev. A 61, 053605 (2000).

[26] M. J. Bijlsma, B. A. Heringa, and H. T. C. Stoof, Phys. Rev. A 61, 053601 (2000).

[27] L. F. Lemmens, F. Brosens, and J. T. Devreese, Phys. Status Solidi B 82, 439 (1977).

[28] J. Tempere and J. T. Devreese, Phys. Rev. B 64, 104504 (2001).

[29] W. Casteels, J. Tempere, and J. T. Devreese, Phys. Rev. A 84, 063612 (2011).

[30] D. H. Santamore and E. Timmermans, New J. Phys. 13, 103029 (2011).

[31] D. C. Roberts and S. Rica, Phys. Rev. Lett. 102, 025301 (2009).

[32] E. Kochetov and M. Smondyrev, Theor. Math. Phys. 85, 1062 (1990).

[33] C. Perroni, G. Iadonisi, and V. Mukhomorov, Eur. Phys. J. B 41, 163 (2004).

[34] M. A. Smondyrev, G. Verbist, F. M. Peeters, and J. T. Devreese, Phys. Rev. B 47, 2596 (1993).

[35] F. Brosens, S. N. Klimin, and J. T. Devreese, Phys. Rev. B 71, 214301 (2005)

[36] R. L. Frank, E. H. Lieb, R. Seiringer, and L. E. Thomas, Phys. Rev. Lett. 104, 210402 (2010).

[37] J. T. Devreese and A. S. Alexandrov, Rep. Prog. Phys. 72, 066501 (2009).

[38] A. S. Alexandrov and N. F. Mott, Rep. Prog. Phys. 57, 1197 (1994)

[39] F. Bassani, M. Geddo, G. Iadonisi, and D. Ninno, Phys. Rev. B 43, 5296 (1991). 
[40] A. S. Alexandrov, Phys. Rev. B 77, 094502 (2008).

[41] A. S. Alexandrov, Theory of Superconductivity: From Weak to Strong Coupling (Taylor and Francis, London, 2003).

[42] G. Verbist, F. M. Peeters, and J. T. Devreese, Phys. Rev. B 43, 2712 (1991).

[43] O. Penrose and L. Onsager, Phys. Rev. 104, 576 (1956).

[44] N. N. Bogoliubov, J. Phys. (USSR) 11, 23 (1947).

[45] L. P. Pitaevskii, Zh. Eksp. Teor. Fiz. 40, 646 (1961).

[46] E. P. Gross, Nuovo Cimento 20, 454 (1961).

[47] A. A. Blinova, M. G. Boshier, and E. Timmermans, arXiv:1304.7704.

[48] R. P. Feynman, Statistical Mechanics: A Set of Lectures (Addison-Wesley, Reading, MA, 1990).

[49] H. Kleinert, Path Integrals in Quantum Mechanics, Statistics, Polymer Physics, and Financial Markets, 5th ed. (World Scientific, Singapore, 2009).

[50] N. N. Bogolubov and S. V. Tyablikov, Zh. Eksp. Teor. Fiz. 19, 256 (1949).
[51] S. I. Pekar, Untersuchungen über die elektronentheorie der kristalle (Akademie Verlag, Berlin, 1951).

[52] N. N. Bogoliubov, in Advances in Theoretical Physics, edited by E. Caianiello (World Scientific, Singapore, 1991), pp. 1-18.

[53] R. Evrard, Phys. Lett. 14, 295 (1965).

[54] W. Casteels, T. Cauteren, J. Tempere, and J. Devreese, Laser Phys. 21, 1480 (2011).

[55] P. Vansant, M. A. Smondyrev, F. M. Peeters, and J. T. Devreese, J. Phys. A 27, 7925 (1994).

[56] M. Bruderer, W. Bao, and D. Jaksch, Europhys. Lett. 82, 30004 (2008).

[57] W. Colson and A. Fetter, J. Low Temp. Phys. 33, 231 (1978).

[58] H.-J. Miesner, D. M. Stamper-Kurn, J. Stenger, S. Inouye, A. P. Chikkatur, and W. Ketterle, Phys. Rev. Lett. 82, 2228 (1999).

[59] P. Ao and S. T. Chui, Phys. Rev. A 58, 4836 (1998).

[60] E. Timmermans, Phys. Rev. Lett. 81, 5718 (1998). 